\title{
Stunned myocardium following acute spinal cord injury: Takotsubo cardiomyopathy after spinal hemorrhage
}

\author{
Chin Ted Chong, FANZCA • Edwin Seet, MMed (Anaes) • \\ Paul Tenenbein, MD
}

Received: 28 November 2009/ Accepted: 6 July 2010/Published online: 19 August 2010

(c) Canadian Anesthesiologists' Society 2010

\section{To the Editor,}

The patient gave written consent for publication of the following case. A 57-yr-old woman presented with sudden onset right flank pain, right leg pain with paralysis, decreased sensation from the waist down, and sharp leftsided chest pain. She had a normal level of consciousness with stable vital signs. The muscle power score in her right lower limb was 0 of 5 . Sensory testing (pain and temperature) showed a decrease below the thoracic spinal nerve 6 (T6), and the electrocardiogram revealed non-specific ST-T wave changes in the anterior leads. The patient's troponin I levels were elevated $\left(2.76 \mu \mathrm{g} \cdot \mathrm{L}^{-1}\right)$, and findings of her chest radiograph were consistent with mild pulmonary edema.

A two-dimensional transthoracic echocardiogram showed left ventricular dysfunction associated with apical and mid-ventricular hypokinesia (Figure). The calculated ejection fraction $(\mathrm{EF})$ was 35\%. Magnetic resonance imaging and spinal angiography confirmed the diagnosis of spinal hemorrhage from a right lateral perimedullary arteriovenous malformation at the T6 level. Urgent surgery was performed involving a thoracic laminectomy and microsurgical resection of the arteriovenous malformation. In anticipation of a possible difficult course of anesthesia due to compromised cardiac function, invasive lines, including arterial and central venous catheters were inserted preoperatively. The patient was hemodynamically stable throughout surgery and did not require inotropic support. Her postoperative recovery was uneventful. A repeat

C. T. Chong, FANZCA $(\bowtie) \cdot$ E. Seet, MMed (Anaes) ·

P. Tenenbein, MD

Toronto Western Hospital, University Health Network,

University of Toronto, Toronto, ON, Canada

e-mail: Chin.Chong@uhn.on.ca echocardiogram two weeks later demonstrated complete recovery of left ventricular function, i.e., a left ventricular ejection fraction (LVEF) of $60 \%$.

This patient had Takotsubo cardiomyopathy, a form of reversible cardiomyopathy characterized by transient dysfunction of the left-ventricle (LV) consisting of apical and mid-ventricular hypokinesia with relative sparing of the basal segment This medical condition creates a configuration on a ventriculogram characteristic of the Japanese octopus trapping pot (tako, "octopus" and tsubo, "pot"). ${ }^{1}$ A unique feature of Takotsubo syndrome is the occurrence of a preceding physical or psychological trigger. Takotsubo syndrome (also known as broken-heart syndrome or transient apical ballooning syndrome) is part of a larger family of cardiomyopathies (now called stress-related cardiomyopathies) that develop in patients with neurogenic myocardial stunning after severe subarachnoid hemorrhage (SAH) or head trauma and in patients with pheochromocytomas, polytrauma, and cocaine abuse. Stress-induced cardiomyopathy has also been described in anaphylaxis during general anesthesia. ${ }^{2}$ It is a condition that may be more frequent in the perioperative setting than commonly appreciated. $^{3}$

As in our patient, Takotsubo syndrome typically occurs in postmenopausal females, often presenting with chest pain at rest. In general, hemodynamic compromise is unusual, but mild to moderate pulmonary edema or congestive heart failure is not uncommon.

What is unusual in our case is the fact that acute spinal injury is implicated in stress-related cardiomyopathy. Acute spinal cord injury has been shown to cause a large increase in systemic blood pressure, albeit unsustained, that is likely secondary to a surge in circulating cathecholamines. ${ }^{4}$ A key hypothesis as to the cause of Takotsubo syndrome is a large increase in circulating catecholamines 


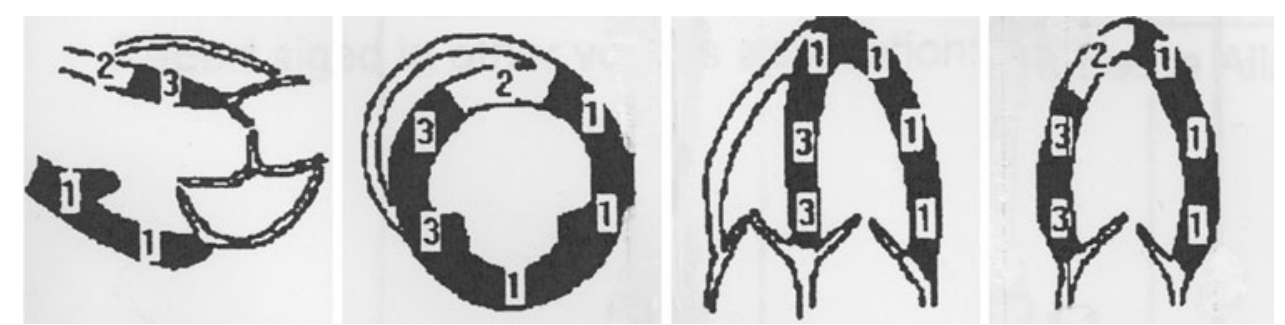

Figure Schematic representations of pre-anesthetic transthoracic echocardiography. Left to right: parasternal long axis, parasternal short axis, apical four-chamber, apical two-chamber views: 1) Normal, 2) Hypokinetic, 3) Akinetic. Note the hypo- and akinetic mid-ventricle and apex

due to either a physical or an emotional stressor. There is a similar pathophysiology of catecholamine cardiotoxicity both in left ventricular dysfunction after SAH and in Takotsubo syndrome. However, the transient reversible LV dysfunction that characterizes Takotsubo syndrome occurs without a necessary concomitant rise in intracranial pressure (unlike SAH).

The way this syndrome mimics myocardial infarction (MI) is well documented..$^{5}$ In the absence of significant cardiovascular risk factors for coronary artery disease, particularly in the perioperative setting of urgent surgery, a high index of awareness of this reversible condition with favourable prognosis could obviate the need for an invasive procedure, e.g., coronary angiography. In this patient, two salient features that favour Takotsubo cardiomyopathy over acute MI are 1) severely reduced EF with large regions of hypokinesa or akinesia accompanied only by modest elevations in troponins, and 2) apical and midventricular wall motion abnormalities that extend beyond a single coronary artery distribution. The widespread use of echocardiography should help to identify this syndrome with its characteristic configuration and rapid resolution in virtually all patients.

Takotsubo cardiomyopathy is recognized increasingly as having a benign course. This finding has repercussions for general anesthesia when urgent surgery is contemplated, such as spinal decompression in our patient. In fact, our patient's hemodynamics remained stable throughout her anesthetic despite an EF of 35\% measured a few days prior. Therefore, similar to cases where patients experience myocardial dysfunction and raised troponins following aneurysmal SAH, we propose that it is generally not necessary to delay surgery to obtain cardiac angiography when the clinical features strongly suggest Takotsubo syndrome.

The overall outcome of patients with myocardial dysfunction following SAH is not limited by cardiac morbidity and likely the same is true in the case of Takotsubo syndrome. Although myocardial dysfunction is associated far more commonly with acute brain injuries, we have described Takotsubo LV dysfunction related to acute spinal injury. The pathophysiology of this phenomenon is likely similar to neurogenic myocardial stunning. The ability to accurately differentiate Takotsubo stress-induced cardiomyopathy from acute MI may become less elusive with increasing awareness amongst anesthesiologists and widespread use of echocardiography in the perioperative setting.

Funding Supported by the Department of Anesthesia, Toronto Western Hospital, University Health Network.

Conflict of interests None declared.

\section{References}

1. Latulippe S, Girard $M$, Denault A. Diagnosing hemodynamic instability in the comatose patient. Can J Anesth 2010; 57: 167-71.

2. Suk EH, Kim DH, Kweon TD, Na SW, Shin JA. Stress-induced cardiomyopathy following cephalosporin-induced anaphylactic shock during general anesthesia. Can J Anesth 2009; 56: 432-6.

3. Bainbridge $D$, Cheng $D$. Stress-induced cardiomyopathy in the perioperative setting. Can J Anesth 2009; 56: 397-401.

4. Guha A, Tator $\mathrm{CH}$. Acute cardiovascular effects of experimental spinal cord injury. J Trauma 1988; 28: 481-90.

5. Bybee KA, Kara T, Prasad A, et al. Systematic review: transient left ventricular apical ballooning: a syndrome that mimics ST segment elevation myocardial infarction. Ann Intern Med 2004; 141: 858-65. 